\title{
Serial studies of pulmonary stenosis in infancy and childhood ${ }^{1}$
}

\author{
Delores Danilowicz, Julien I. E. Hoffman, and Abraham M. Rudolph \\ From the Department of Pediatrics, New York University, New York, NY, and the Department of Pediatrics \\ and the Cardiovascular Research Institute, University of California-San Francisco; San Francisco, California, \\ U.S.A.
}

Thirty-five children with pulmonary stenosis were catheterized from I day to 9 years of age and recatheterized after 2 weeks to 15 years. Right ventricular systolic pressure rose in 24 and the increase was greater in those under than over 5 years old. Pulmonary valve orifice area per square metre of body surface area increased in 12, did not change in 3, and fell in 17; absolute decrease in calculated orifice area was usually associated with infundibular hypertrophy. Increase in right ventricular systolic pressure with age was thus caused by failure of the valve orifice to grow fast enough to keep pace with the increase of stroke volume.

In the whole group, increasingly severe pulmonary stenosis was matched by increasing electrocardiographic evidence of right ventricular hypertrophy. However, in individual patients the electrocardiogram could suggest that right ventricular pressure had decreased when in fact it had risen considerably.

Severe pulmonary stenosis may cause congestive heart failure and death in infancy or early childhood (Benton et al., 1962; Luke, 1966; Gersony et al., 1967). However, relatively few of those born with pulmonary stenosis follow this course (Carlgren, 1959; Rowe and Cleary, 1960; Lambert, Canent, and Hohn, 1966) and the majority of deaths have occurred in older children and adults (Selzer et al., 1949; Abrahams and Wood, 1951; Fabricius, 1959). Most of the reported older patients with severe pulmonary stenosis did not have serial cardiac catheterizations and we do not know if they had from infancy a tight stenosis that was well tolerated for many years, or if the obstruction was mild in infancy and became more severe with time.

There have been few serial catheterization studies of the natural history of pulmonary stenosis in children and even fewer in very young children. Out of a total of 105 recatheterized patients cited in 8 reports (Fabricius, 1959; Engle, Ito, and Goldberg, 1964; Tinker et al., 1965; Levine and Blumenthal, 1965; Moller and Adams, 1965; Loogan, Gleichmann, and Wilke, 1966; Lueker, Vogel, and Blount, 1970; Møller, Wennevold, and Lyngborg, 1973), the right ventricular pressure had risen significantly in

Received 13 March 1975.

${ }^{1}$ Supported in part by a program project grant from the National Heart and Lung Institute, National Institutes of Health, Bethesda, Maryland, U.S.A. the second study in only 15, almost all of whom had high pressures at the first study. Furthermore, most of the patients were older children and adults; only 15 were under 5 years at the time of the first study and none were infants. The general lack of change of right ventricular pressure with time suggests that the pulmonary valve area increased with age in most of these patients, and this was confirmed by calculations of the valve area in 2 of these studies (Moller and Adams, 1965; Lueker et al., 1970). While this information is important it does not throw any light on the changes occurring in the few with increasingly severe disease.

There are several ways in which pulmonary stenosis might become more severe. Some studies (Kirklin et al., 1953; Johnson, 1959; Little, Lavender, and DeSanctis, 1963) have suggested progressive obstruction of the right ventricular outflow tract from increasing hypertrophy of infundibular muscle but these have all been in older children and adults. Campbell and Missen (1969) speculated that fibrin deposited on a stenotic valve could diminish the orifice area. Finally, Engle and Taussig (1950) considered that the orifice of a stenotic pulmonary valve might remain the same size and so become relatively smaller as body size and cardiac output increased with growth. This might explain why Tinker et al. (1965) noted that, when there was electrocardiographic evidence of deterioration in 
patients with pulmonary stenosis, it usually took place during the adolescent growth spurt.

Because of the lack of information about the changes that take place in pulmonary stenosis in younger children, we here report serial clinical and cardiac catheterization studies of 35 infants and young children with congenital pulmonary stenosis.

\section{Subjects and methods}

Thirty-five children were first catheterized between I day and 9 years of age, and recatheterized at intervals ranging from 2 weeks to 15 years; 6 children had third catheterizations. Twelve were seen at the Bronx Municipal Hospital Center or Lincoln Hospital in New York, I3 at the New York University Medical Center, and Io at the University of California-San Francisco. One of the I7 girls and I boy had Noonan's syndrome.

All had valvar pulmonary stenosis with an intact ventricular septum. Two with rubella embryopathy had added mild peripheral pulmonary arterial stenosis. Three had small left-to-right shunts with pulmonary to systemic flow ratios of $I: 8,1: 3$, and $I: 2$; one shunt was at the atrial level, one through a persistent ductus, and one at both levels.

No sedation or anaesthesia was used for 8 infants catheterized under 3 months of age; ro other infants received general anaesthesia $(5)$ or sedation with tribromoethyl alcohol (3), morphine sulphate (I), or chloral hydrate (I). The remainder were usually premedicated with pethidine and hydroxyzine hydrochloride, each I $\mathrm{mg} / \mathrm{kg}$ intramuscularly, and were never deeply sedated though they were usually quiet throughout the study.

Oxygen uptake was measured in 3 infants with a nasal valve (Golinko and Rudolph, 196r) and in most over 7 years of age by collection of expired air. For the others, oxygen uptake was estimated from average values for heart rate and body surface area (Hoffman and Rudolph, 1965; LaFarge and Miettinen, 1970); the 95 per cent confidence limits for these assumed values are about 50 per cent each side of the mean value. Oxygen saturations were measured spectrophotometrically by a modified Drabkin method (Rudolph and Cayler, 1958) or, more recently, by reflectance oximetry. Flows were calculated by the Fick principle. Whenever possible, pressures in the pulmonary artery and right ventricle were measured using a catheter with one or more side holes. The reference level for all pressures was the midchest.

The area of the pulmonary valve orifice was estimated by the formula of Gorlin and Gorlin (195I); the flow across the valve was the pulmonary blood flow in all but the patient with the ductal shunt, in whom systemic flow was used. When there was infundibular stenosis, the same formula was used to indicate effective orifice area. In some infants catheterized at other hospitals, the pressure tracings were not available for calculating systolic ejection time and the mean pressure difference from right ventricle to pulmonary artery. We then used the formula of Bassingthwaighte et al. (1963) to derive the mean systolic pressure difference, or the formula of Moller and Adams (1966) to calculate pulmonary valve area from pulmonary valvar resistance.
At the first cardiac catheterization, 5 children (I a neonate) had peak right ventricular systolic pressures at or under $35 \mathrm{mmHg}(4.7 \mathrm{kPa})$; the mean systolic pressure differences across the pulmonary valves ranged from 6 to $18 \mathrm{mmHg}$ ( 0.8 to $2.4 \mathrm{kPa}$ ). All had thickened but mobile pulmonary valves seen at angiography and none had more than mild dilatation of the main pulmonary artery. One of the 2 children with Noonan's syndrome had a thickened, myxomatous valve at subsequent surgery, while the other had no angiographic evidence of this change. Angiography was also used to assess qualitatively the presence and degree of infundibular hypertrophy.

\section{Results}

The pertinent clinical and catheterization data are given in Table I. Fig. I shows how the right ventricular systolic pressure changed at subsequent catheterizations and, since pulmonary arterial pressures in pulmonary stenosis do not vary much, also represents the changes in peak systolic pressure difference across the right ventricular outflow tract. There was a tendency for the right ventricular systolic pressure to rise, especially in younger children and those with higher initial pressures. Of 25 first catheterized under 5 years of age, pressure rose in 17 (over $20 \mathrm{mmHg}(2.7 \mathrm{kPa}$ ) in II), fell in 6 , and rose and then fell in 2 . The falls of pressure were under $20 \mathrm{mmHg}(2.7 \mathrm{kPa})$ in all but 2 children, in I of whom a left-to-right atrial shunt disappeared spontaneously between the 2 studies. A systolic pressure rise over $20 \mathrm{mmHg}(2.7 \mathrm{kPa})$ was noted in 2 out of 7 and in 7 out of 12 whose initial right ventricular systolic pressures were, respectively, under 50 and between $5 \mathrm{I}$ and $100 \mathrm{mmHg}(6.7$ and 13.3 $\mathrm{kPa})$. The difference was not significant $(P=0.18)$ by Fisher's exact test. Of Io children first catheterized over 5 years of age, right ventricular systolic pressure rose in 7 (in 4 it rose less than $20 \mathrm{mmHg}$ $(2.7 \mathrm{kPa})$ ) and fell less than $10 \mathrm{mmHg}(1.2 \mathrm{kPa})$ in 3 . The smaller rise of pressure in those first studied over 5 years is all the more striking because they usually had the greater time intervals between catheterizations.

A major cause of a change in right ventricular systolic pressure between studies is a change in stroke volume; this usually increased with age and growth and could have been increased or decreased by differences in sedation and anxiety. The two variables tended to rise together but could at times vary in opposite directions (Fig. 2).

We measured changes in right ventricular systolic ejection time in 23 patients; in 7 there was a slight decrease at the second study of 0.01 to $0.08 \mathrm{~s}$, in 3 there was no change, and in 13 ejection time increased up to $0.19 \mathrm{~s}$, with a mean increase of $0.066 \mathrm{~s}$. Only 3 of the 23 measurements had ratios of ejection 
TABLE I Serial catheterization and electrocardiographic data

\begin{tabular}{|c|c|c|c|c|c|c|c|c|c|c|c|}
\hline \multirow{2}{*}{$\begin{array}{l}\text { Case } \\
\text { No. }\end{array}$} & \multirow[t]{2}{*}{ Age } & \multirow[t]{2}{*}{$B S A\left(m^{2}\right)$} & \multicolumn{4}{|c|}{ Pressures $(\mathrm{mmHg})$} & \multirow{2}{*}{$\begin{array}{c}\mathrm{SaO} \mathrm{O}_{2} \\
(\%)\end{array}$} & \multirow{2}{*}{$\begin{array}{l}A V \mathrm{O}_{2} \\
\text { difference } \\
(m l / l)\end{array}$} & \multirow{2}{*}{$\begin{array}{l}Q v \\
\left(l / \min \text { per } m^{2}\right)\end{array}$} & \multirow[t]{2}{*}{$H R$} & \multirow[t]{2}{*}{$S V(m l)$} \\
\hline & & & $a$ & $\begin{array}{l}R A \\
\text { mean }\end{array}$ & $R V$ & $M P A$ & & & & & \\
\hline & $\int I d$ & 0.18 & Io & 8 & $72 / 6$ & $58 / 44$ & & 28 & 4.7 & 180 & 5 \\
\hline I & $\left\{\begin{array}{l}18 d \\
64 d\end{array}\right.$ & $\begin{array}{l}0.18 \\
0.23\end{array}$ & 6 & 4 & $\begin{array}{r}96 / 6 \\
100 / 6\end{array}$ & $\begin{array}{l}78 / 22 \\
52 / 29\end{array}$ & & 24 & 5.8 & $\begin{array}{l}180 \\
120\end{array}$ & II \\
\hline 2 & $\left\{\begin{array}{rl}4 & d \\
18 & d \\
I & y r\end{array}\right.$ & $\begin{array}{l}0.11 \\
0.11 \\
0.40\end{array}$ & $\begin{array}{r}0 \\
2 \\
14\end{array}$ & $\begin{array}{r}-2 \\
-2 \\
7\end{array}$ & $\begin{array}{l}72 /- \\
102 /- \\
200 / 6\end{array}$ & $16 / 8$ & $\begin{array}{l}89 \\
86 \\
89\end{array}$ & $\begin{array}{l}61 \\
48 \\
58\end{array}$ & $\begin{array}{l}2.6 \\
3.2 \\
2.4\end{array}$ & $\begin{array}{l}160 \\
140 \\
108\end{array}$ & $\begin{array}{l}1.8 \\
2.5 \\
9\end{array}$ \\
\hline 3 & $\left\{\begin{array}{l}5 \mathrm{~d} \\
\mathrm{I} \text { yr } 2 \mathrm{~m}\end{array}\right.$ & $\begin{array}{l}0.16 \\
0.44\end{array}$ & $\begin{array}{l}\text { I } \\
6\end{array}$ & $\begin{array}{l}0 \\
3\end{array}$ & $\begin{array}{l}32 / 0 \\
75 / 6\end{array}$ & $\begin{array}{l}26 / 7 \\
37 / 8\end{array}$ & $\begin{array}{l}97 \\
99\end{array}$ & $\begin{array}{l}22 \\
30\end{array}$ & $\begin{array}{l}3.9 \\
5.0\end{array}$ & $\begin{array}{l}150 \\
120\end{array}$ & $\begin{array}{r}4 \\
18\end{array}$ \\
\hline 4 & $\left\{\begin{aligned} \text { I0 d } \\
\text { I yr } 4 \mathrm{~m}\end{aligned}\right.$ & $\begin{array}{l}0.19 \\
0.42\end{array}$ & $\begin{array}{l}6 \\
8\end{array}$ & $\begin{array}{l}x \\
4\end{array}$ & $\begin{array}{l}95 / 6 \\
45 / 6\end{array}$ & $\begin{array}{l}32 / 10 \\
24 / 12\end{array}$ & $\begin{array}{l}94 \\
97\end{array}$ & $\begin{array}{l}33 \\
41\end{array}$ & $\begin{array}{l}4.0 \\
3.2\end{array}$ & $\begin{array}{l}160 \\
120\end{array}$ & $\begin{array}{l}4.8 \\
\text { I }\end{array}$ \\
\hline 5 & $\begin{array}{l}3 \mathrm{w} \\
\mathrm{I} \mathrm{yr} \mathrm{I} \mathrm{m}\end{array}$ & $\begin{array}{l}0.23 \\
0.46\end{array}$ & $\begin{array}{l}2 \\
8\end{array}$ & $\begin{array}{l}I \\
5\end{array}$ & $\begin{array}{l}38 /- \\
50 / 7\end{array}$ & $\begin{array}{l}22 /- \\
24 / 16\end{array}$ & 95 & $\begin{array}{l}20 \\
17\end{array}$ & $\begin{array}{l}7.5 \\
5.4\end{array}$ & $\begin{array}{l}120 \\
120\end{array}$ & $\begin{array}{l}14 \\
21\end{array}$ \\
\hline 6 & $\begin{array}{l}6 \mathrm{w} \\
4 \mathrm{~m}\end{array}$ & $\begin{array}{l}0.27 \\
0.33\end{array}$ & $\begin{array}{l}\text { II } \\
\text { I2 }\end{array}$ & $\begin{array}{l}6 \\
5\end{array}$ & $\begin{array}{l}190 / 10 \\
175 / 8\end{array}$ & & $\begin{array}{l}98 \\
98\end{array}$ & $\begin{array}{l}22 \\
43\end{array}$ & $\begin{array}{l}5.9 \\
3.0\end{array}$ & $\begin{array}{l}150 \\
150\end{array}$ & $\begin{array}{r}\text { II } \\
7\end{array}$ \\
\hline 7 & $\begin{array}{l}2 \frac{1}{2} \mathrm{~m} \\
10 \mathrm{~m} \\
2 \mathrm{yr} 6 \mathrm{~m}\end{array}$ & $\begin{array}{l}0.30 \\
0.47 \\
0.58\end{array}$ & 4 & $\mathbf{I}$ & $\begin{array}{c}70 /- \\
106 /- \\
75 / 8\end{array}$ & $\begin{array}{l}9 / 5 \\
16 / 4 \\
23 / 14\end{array}$ & $\begin{array}{l}95 \\
95 \\
98\end{array}$ & $\begin{array}{l}44 \\
24 \\
25\end{array}$ & $\begin{array}{l}3.2 \\
6.2 \\
6.0\end{array}$ & $\begin{array}{l}150 \\
120 \\
140\end{array}$ & $\begin{array}{r}6 \\
24 \\
25\end{array}$ \\
\hline 8 & $\begin{array}{l}4 \mathrm{~m} \\
\mathrm{I} \mathrm{yr} \frac{1}{2} \mathrm{~m} \\
3 \mathrm{yr}\end{array}$ & $\begin{array}{l}0.31 \\
0.55 \\
0.80\end{array}$ & $\begin{array}{r}8 \\
\text { II }\end{array}$ & $\begin{array}{l}6 \\
8\end{array}$ & $\begin{array}{c}76 / 10 \\
110 / 12 \\
60 / 3\end{array}$ & $\begin{array}{l}25 / 15 \\
30 / 19 \\
24 / 5\end{array}$ & & $\begin{array}{l}35 \\
20\end{array}$ & $\begin{array}{l}3.7 \\
7.5\end{array}$ & $\begin{array}{r}150 \\
120 \\
90\end{array}$ & $\begin{array}{r}8 \\
34\end{array}$ \\
\hline 9 & $\begin{array}{l}5 \mathrm{~m} \\
3 \mathrm{yr}\end{array}$ & $\begin{array}{l}0.40 \\
0.54\end{array}$ & $\begin{array}{r}3 \\
10\end{array}$ & $\begin{array}{l}1 \\
8\end{array}$ & $\begin{array}{l}38 / 1 \\
50 / 6\end{array}$ & $\begin{array}{l}25 / 9 \\
12 / 6\end{array}$ & 93 & 23 & 6.5 & $\begin{array}{l}150 \\
100\end{array}$ & 35 \\
\hline 10 & $\begin{array}{l}5 \mathrm{~m} \\
\mathrm{I} \mathrm{yr} 5 \mathrm{~m}\end{array}$ & $\begin{array}{l}0.32 \\
0.52\end{array}$ & $\begin{array}{l}6 \\
7\end{array}$ & $\begin{array}{l}5 \\
4\end{array}$ & $\begin{array}{r}85 / 4 \\
100 / 6\end{array}$ & $\begin{array}{l}20 / 10 \\
16 / 8\end{array}$ & $\begin{array}{l}93 \\
94\end{array}$ & $\begin{array}{l}36 \\
40\end{array}$ & $\begin{array}{l}3.6 \\
4.1\end{array}$ & $\begin{array}{l}136 \\
116\end{array}$ & $\begin{array}{l}8.5 \\
18\end{array}$ \\
\hline II & $\begin{array}{l}5 \mathrm{~m} \\
\mathrm{I} \mathrm{yr} 4 \mathrm{~m} \\
\text { I yr I I m }\end{array}$ & $\begin{array}{r}0.41 \\
0.52 \\
0.55\end{array}$ & $\begin{array}{r}7 \\
\text { II } \\
\text { I4 }\end{array}$ & $\begin{array}{l}4 \\
6 \\
8\end{array}$ & $\begin{array}{l}104 / 6 \\
130 / 8 \\
192 / 12\end{array}$ & $\begin{array}{l}16 / 8 \\
14 / 4 \\
17 / 13\end{array}$ & $\begin{array}{l}96 \\
96 \\
97\end{array}$ & $\begin{array}{l}43 \\
40 \\
66\end{array}$ & $\begin{array}{l}3.5 \\
3.7 \\
2.3\end{array}$ & $\begin{array}{l}115 \\
120 \\
120\end{array}$ & $\begin{array}{l}12 \\
16 \\
11\end{array}$ \\
\hline 12 & $\begin{array}{l}6 \mathrm{~m} \\
10 \mathrm{~m}\end{array}$ & $\begin{array}{l}0.45 \\
0.40\end{array}$ & Io & 4 & $\begin{array}{l}110 / 4 \\
140 / 8\end{array}$ & & 92 & 78.5 & 1.9 & 150 & 5 \\
\hline 13 & $\begin{array}{l}7 \mathrm{~m} \\
2 \mathrm{yr} 2 \mathrm{~m}\end{array}$ & $\begin{array}{l}0.25 \\
0.50\end{array}$ & IO & $\begin{array}{l}4 \\
7\end{array}$ & $\begin{array}{r}82 / 8 \\
160 / 6\end{array}$ & $\begin{array}{l}16 / 10 \\
13 / 8\end{array}$ & $\begin{array}{l}90 \\
93\end{array}$ & $\begin{array}{l}27 \\
38\end{array}$ & $\begin{array}{l}5.6 \\
4.0\end{array}$ & $\begin{array}{l}\text { I44 } \\
\text { IIO }\end{array}$ & $\begin{array}{l}10 \\
18\end{array}$ \\
\hline 14 & $\begin{array}{l}\text { I yr } \\
4 \mathrm{yr}\end{array}$ & $\begin{array}{l}0.56 \\
0.70\end{array}$ & $\begin{array}{l}8 \\
8\end{array}$ & $\begin{array}{l}6 \\
6\end{array}$ & $\begin{array}{l}59 / 8 \\
80 / 10\end{array}$ & $\begin{array}{l}22 / 12 \\
18 / 10\end{array}$ & $\begin{array}{l}96 \\
93\end{array}$ & $\begin{array}{l}2 I \\
44.5\end{array}$ & $\begin{array}{l}7.1 \\
3 \cdot 4\end{array}$ & $\begin{array}{l}140 \\
100\end{array}$ & $\begin{array}{l}28 \\
24\end{array}$ \\
\hline 15 & $\begin{array}{l}\text { I yr } 4 \mathrm{~m} \\
2 \mathrm{yr} \mathrm{I} \mathrm{m} \\
4 \mathrm{yr}\end{array}$ & $\begin{array}{l}0.49 \\
0.49 \\
0.81\end{array}$ & $\begin{array}{l}2 \\
5\end{array}$ & $\begin{array}{l}I \\
4\end{array}$ & $\begin{array}{l}\text { Iro/ro } \\
90 / 10 \\
83 / 8\end{array}$ & $\begin{array}{l}16 / 9 \\
15 / 8 \\
20 / 11\end{array}$ & $\begin{array}{l}93 \\
95\end{array}$ & $\begin{array}{l}37 \\
42\end{array}$ & $\begin{array}{l}4.3 \\
3.6\end{array}$ & $\begin{array}{l}160 \\
120 \\
100\end{array}$ & $\begin{array}{l}13 \\
15\end{array}$ \\
\hline I6 & $\begin{array}{l}\mathrm{I} \mathrm{yr} 6 \mathrm{~m} \\
4 \mathrm{yr} 6 \mathrm{~m}\end{array}$ & $\begin{array}{l}0.42 \\
0.68\end{array}$ & & & $\begin{array}{l}40 / 2 \\
55 / 8\end{array}$ & $\begin{array}{l}16 / 6 \\
14 / 10\end{array}$ & & $\begin{array}{l}28 \\
28 \cdot 5\end{array}$ & $5 \cdot 3$ & IIO & 33 \\
\hline I7 & $\begin{array}{l}2 \mathrm{yr} 4 \mathrm{~m} \\
3 \mathrm{yr}\end{array}$ & $\begin{array}{l}0.58 \\
0.58\end{array}$ & $\begin{array}{r}5 \\
14\end{array}$ & $\begin{array}{l}4 \\
8\end{array}$ & $\begin{array}{l}145 / 6 \\
130 / 12\end{array}$ & $16 / 8$ & $\begin{array}{l}85 \\
79\end{array}$ & $\begin{array}{l}78 \\
60\end{array}$ & $\begin{array}{l}1.9 \\
2.5\end{array}$ & $\begin{array}{l}140 \\
120\end{array}$ & $\begin{array}{r}8 \\
12\end{array}$ \\
\hline 18 & $\begin{array}{l}2 \mathrm{yr} 6 \mathrm{~m} \\
4 \mathrm{yr}\end{array}$ & $\begin{array}{l}0.58 \\
0.67\end{array}$ & $\begin{array}{l}\text { I0 } \\
\text { 10 }\end{array}$ & $\begin{array}{l}3 \\
5\end{array}$ & $\begin{array}{l}102 / 6 \\
120 / 6\end{array}$ & $\begin{array}{l}14 / 9 \\
17 / 10\end{array}$ & $\begin{array}{l}97 \\
96\end{array}$ & $\begin{array}{l}32 \\
32\end{array}$ & $\begin{array}{l}4 \cdot 7 \\
4 \cdot 7\end{array}$ & $\begin{array}{l}125 \\
135\end{array}$ & $\begin{array}{l}22 \\
23\end{array}$ \\
\hline I9 & $\begin{array}{l}3 \mathrm{yr} \\
5 \mathrm{yr}\end{array}$ & $\begin{array}{l}0.64 \\
0.80\end{array}$ & $\begin{array}{l}0 \\
7\end{array}$ & $\begin{array}{l}2 \\
2\end{array}$ & $\begin{array}{c}76 / 6 \\
108 / 12\end{array}$ & $\begin{array}{l}15 / 10 \\
23 / 10\end{array}$ & $\begin{array}{l}96 \\
95\end{array}$ & $\begin{array}{l}46.5 \\
37.5\end{array}$ & $\begin{array}{l}3.6 \\
3 \cdot 7\end{array}$ & $\begin{array}{r}120 \\
75\end{array}$ & $\begin{array}{l}19 \\
40\end{array}$ \\
\hline 20 & $\left\{\begin{array}{l}3 \mathrm{yr} \\
6 \mathrm{yr}\end{array}\right.$ & $\begin{array}{l}0.62 \\
0.83\end{array}$ & $\begin{array}{l}5 \\
8\end{array}$ & $\begin{array}{l}3 \\
4\end{array}$ & $\begin{array}{c}48 / 6 \\
100 / 12\end{array}$ & $\begin{array}{l}18 / 8 \\
22 / 10\end{array}$ & $\begin{array}{l}98 \\
92\end{array}$ & $\begin{array}{l}32 \\
32\end{array}$ & $\begin{array}{l}5.0 \\
4.8\end{array}$ & $\begin{array}{l}96 \\
96\end{array}$ & $\begin{array}{l}32 \\
42\end{array}$ \\
\hline $2 \mathrm{I}$ & $\left\{\begin{array}{l}3 \text { yr } \\
8 \text { yr Ir m }\end{array}\right.$ & $\begin{array}{l}0.55 \\
0.90\end{array}$ & $\begin{array}{r}8 \\
\text { II }\end{array}$ & $\begin{array}{l}7 \\
8\end{array}$ & $\begin{array}{l}63 / 10 \\
75 / 11\end{array}$ & $\begin{array}{l}22 / 10 \\
22 / 10\end{array}$ & 98 & $\begin{array}{l}44 \\
37\end{array}$ & $\begin{array}{l}3.5 \\
4.0\end{array}$ & $\begin{array}{l}90 \\
80\end{array}$ & $\begin{array}{l}21 \\
45\end{array}$ \\
\hline 22 & $\left\{\begin{array}{r}3 \mathrm{yr} \\
10 \mathrm{yr}\end{array}\right.$ & $\begin{array}{l}0.70 \\
1.60\end{array}$ & 3 & & $\begin{array}{l}32 / 6 \\
23 / 3\end{array}$ & $\begin{array}{l}21 / 8 \\
13 / 5\end{array}$ & 96 & 38 & 3.8 & 85 & 72 \\
\hline 23 & $\left\{\begin{array}{l}3 \mathrm{yr} 6 \mathrm{~m} \\
\mathrm{IO} \mathrm{yr}\end{array}\right.$ & $\begin{array}{l}0.65 \\
1.12\end{array}$ & $\begin{array}{l}7 \\
8\end{array}$ & $\begin{array}{l}5 \\
7\end{array}$ & $\begin{array}{c}80 / 7 \\
105 / 14\end{array}$ & $\begin{array}{l}27 / 9 \\
28 / 10\end{array}$ & $\begin{array}{l}99 \\
98\end{array}$ & $\begin{array}{l}44 \\
50\end{array}$ & $\begin{array}{l}3.4 \\
2.7\end{array}$ & $\begin{array}{l}94 \\
86\end{array}$ & $\begin{array}{l}24 \\
35\end{array}$ \\
\hline
\end{tabular}




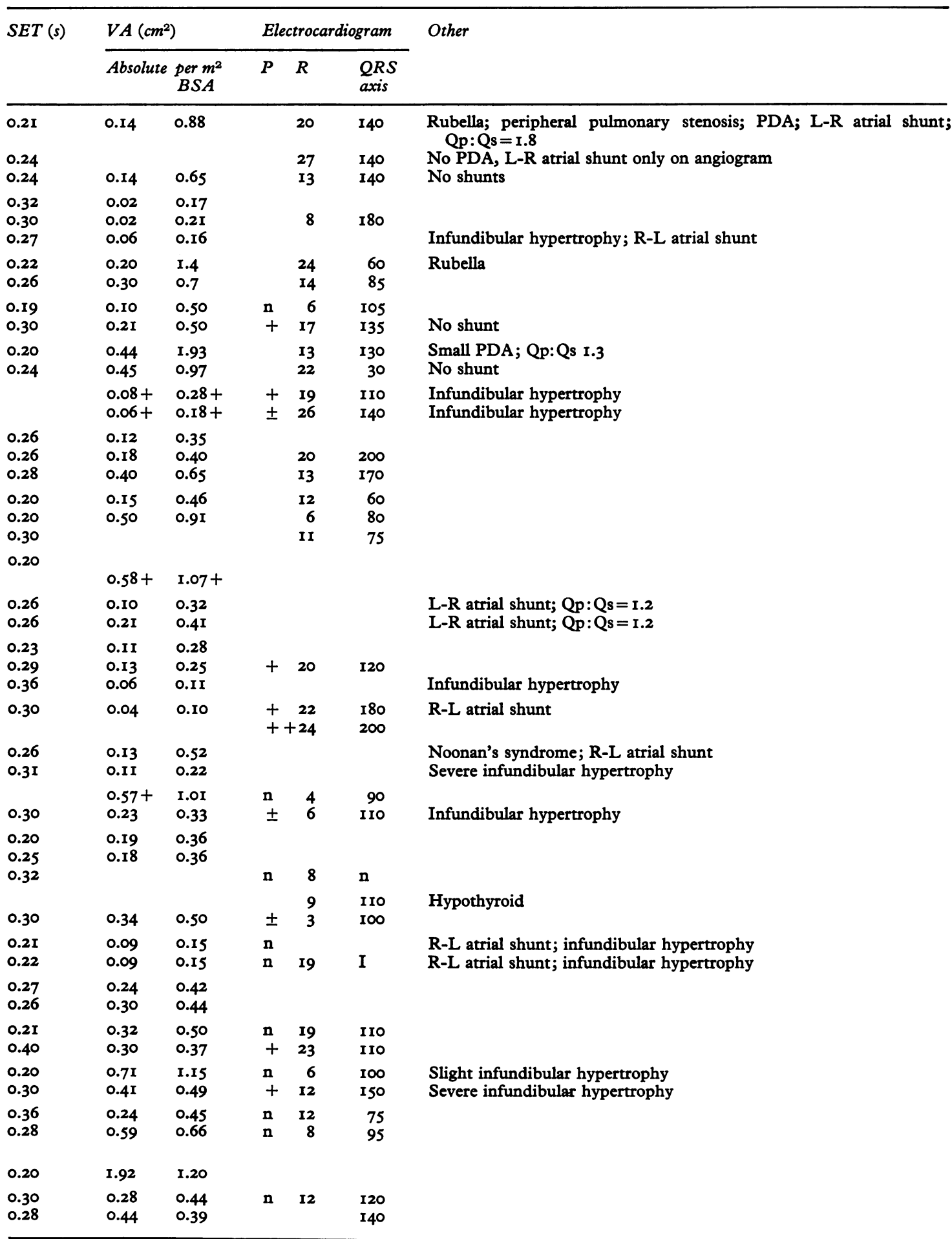


TABLE I (Cont'd)

\begin{tabular}{|c|c|c|c|c|c|c|c|c|c|c|c|}
\hline \multirow{2}{*}{$\begin{array}{l}\text { Case } \\
\text { No. }\end{array}$} & \multirow[t]{2}{*}{ Age } & \multirow[t]{2}{*}{$B S A\left(m^{2}\right)$} & \multicolumn{4}{|c|}{ Pressures $(\mathrm{mmHg})$} & \multirow{2}{*}{$\mathrm{SaO}_{2} \%$} & \multirow{2}{*}{$\begin{array}{c}A V \mathrm{O}_{2} \\
\text { difference } \\
(\mathrm{ml} / \mathrm{l})\end{array}$} & \multirow{2}{*}{$\begin{array}{l}Q v \\
\left(l / \min \text { per } m^{2}\right)\end{array}$} & \multirow[t]{2}{*}{$H R$} & \multirow[t]{2}{*}{$S V(m l)$} \\
\hline & & & $a$ & $\begin{array}{c}R A \\
\text { mean }\end{array}$ & $R V$ & $M P A$ & & & & & \\
\hline 24 & $\begin{array}{l}4 \mathrm{yr} \\
\mathrm{r} 4 \mathrm{yr} 6 \mathrm{~m}\end{array}$ & $\begin{array}{l}0.68 \\
1.70\end{array}$ & 12 & 5 & $\begin{array}{l}54 / 3 \\
90 / 10\end{array}$ & $\begin{array}{l}13 / 6 \\
20 / 10\end{array}$ & $\begin{array}{l}98 \\
95\end{array}$ & $\begin{array}{l}29 \\
44\end{array}$ & $\begin{array}{l}5.5 \\
3.6\end{array}$ & $\begin{array}{r}100 \\
72\end{array}$ & $\begin{array}{l}37 \\
85\end{array}$ \\
\hline 25 & $\begin{array}{r}4 \mathrm{yr} 9 \mathrm{~m} \\
\mathrm{I} 3 \mathrm{yr} 9 \mathrm{~m}\end{array}$ & $\begin{array}{l}0.76 \\
1.42\end{array}$ & $\begin{array}{l}7 \\
8\end{array}$ & $\begin{array}{l}3 \\
4\end{array}$ & $\begin{array}{l}35 / 8 \\
32 / 8\end{array}$ & $\begin{array}{l}20 / 7 \\
22 / 8\end{array}$ & $\begin{array}{l}95 \\
95\end{array}$ & $\begin{array}{l}41 \\
50\end{array}$ & $\begin{array}{l}3.8 \\
3.4\end{array}$ & $\begin{array}{l}82 \\
85\end{array}$ & $\begin{array}{l}35 \\
57\end{array}$ \\
\hline 26 & $\begin{array}{l}5 \mathrm{yr} \\
10 \mathrm{yr}\end{array}$ & $\begin{array}{l}0.75 \\
1.18\end{array}$ & $\begin{array}{r}\text { 10 } \\
7\end{array}$ & $\begin{array}{l}2 \\
3\end{array}$ & $\begin{array}{l}80 / 6 \\
95 / 8\end{array}$ & $\begin{array}{l}25 / 6 \\
18 / 6\end{array}$ & $\begin{array}{l}97 \\
95\end{array}$ & $\begin{array}{l}39 \\
29\end{array}$ & $\begin{array}{l}4.1 \\
5.2\end{array}$ & $\begin{array}{r}100 \\
80\end{array}$ & $\begin{array}{l}30 \\
76\end{array}$ \\
\hline 27 & $\begin{array}{l}5 \mathrm{yr} 7 \mathrm{~m} \\
\mathrm{r} 3 \mathrm{yr}\end{array}$ & $\begin{array}{l}0.79 \\
1.43\end{array}$ & 7 & $\begin{array}{l}5 \\
5\end{array}$ & $\begin{array}{l}60 / 5 \\
55 / 9\end{array}$ & $\begin{array}{l}15 / 10 \\
18 / 8\end{array}$ & $\begin{array}{l}98 \\
94\end{array}$ & $\begin{array}{l}39 \\
4 I\end{array}$ & $\begin{array}{l}4.1 \\
5.1\end{array}$ & $\begin{array}{r}120 \\
70\end{array}$ & $\begin{array}{r}27 \\
105\end{array}$ \\
\hline 28 & $\begin{array}{l}6 \mathrm{yr} \\
8 \mathrm{yr}\end{array}$ & $\begin{array}{l}0.80 \\
0.95\end{array}$ & $\begin{array}{r}6 \\
11\end{array}$ & $\begin{array}{l}3 \\
6\end{array}$ & $\begin{array}{r}60 / 6 \\
100 / 8\end{array}$ & $\begin{array}{l}26 / 8 \\
19 / 7\end{array}$ & $\begin{array}{l}95 \\
94\end{array}$ & $\begin{array}{l}24 \\
37\end{array}$ & $\begin{array}{l}6.9 \\
4.1\end{array}$ & $\begin{array}{l}120 \\
100\end{array}$ & $\begin{array}{l}46 \\
39\end{array}$ \\
\hline 29 & $\begin{array}{r}6 \mathrm{yr} \\
14 \mathrm{yr}\end{array}$ & $\begin{array}{l}0.78 \\
1.29\end{array}$ & 7 & 3 & $\begin{array}{l}66 / 9 \\
80 / 9\end{array}$ & $\begin{array}{l}20 / 9 \\
20 / 10\end{array}$ & $\begin{array}{l}96 \\
99\end{array}$ & $\begin{array}{l}44 \\
63\end{array}$ & $\begin{array}{l}3.6 \\
2.3\end{array}$ & $\begin{array}{r}100 \\
70\end{array}$ & $\begin{array}{l}28 \\
42\end{array}$ \\
\hline 30 & $\begin{array}{r}6 \mathrm{yr} \\
17 \mathrm{yr}\end{array}$ & $\begin{array}{l}0.90 \\
1.75\end{array}$ & 4 & 3 & $\begin{array}{l}19 / 0 \\
25 / 4\end{array}$ & $\begin{array}{l}11 / 3 \\
15 / 5\end{array}$ & 95 & 40 & 3.4 & 80 & 74 \\
\hline 31 & $\begin{array}{l}6 \mathrm{yr} 3 \mathrm{~m} \\
13 \mathrm{yr}\end{array}$ & $\begin{array}{l}0.86 \\
1.40\end{array}$ & 7 & 3 & $\begin{array}{l}48 / 2 \\
75 / 7\end{array}$ & $\begin{array}{l}12 / 5 \\
15 / 5\end{array}$ & 98 & 46 & 2.6 & 60 & 60 \\
\hline 32 & $\left\{\begin{array}{rl}7 & \mathrm{yr} \\
12 & \mathrm{yr}\end{array}\right.$ & $\begin{array}{l}0.80 \\
1.40\end{array}$ & $\begin{array}{l}9 \\
3\end{array}$ & $\begin{array}{l}5 \\
1\end{array}$ & $\begin{array}{l}60 / 10 \\
80 / 4\end{array}$ & $\begin{array}{l}31 / 18 \\
18 / 12\end{array}$ & $\begin{array}{l}94 \\
94\end{array}$ & 35 & $\begin{array}{l}4.5 \\
4.6\end{array}$ & $\begin{array}{r}110 \\
90\end{array}$ & $\begin{array}{l}33 \\
72\end{array}$ \\
\hline 33 & $\left\{\begin{array}{r}7 \mathrm{yr} \\
17 \mathrm{yr}\end{array}\right.$ & $\begin{array}{l}0.96 \\
1.82\end{array}$ & II & I & $\begin{array}{l}29 / 1 \\
21 / 4\end{array}$ & $\begin{array}{l}11 / 6 \\
12 / 5\end{array}$ & 96 & 46 & $\begin{array}{l}3.5 \\
2.9\end{array}$ & $\begin{array}{r}114 \\
75\end{array}$ & $\begin{array}{l}23 \\
70\end{array}$ \\
\hline 34 & $\left\{\begin{array}{r}7 \mathrm{yr} \\
18 \mathrm{yr}\end{array}\right.$ & $\begin{array}{l}0.74 \\
1.42\end{array}$ & $\begin{array}{r}6 \\
\text { II }\end{array}$ & $\begin{array}{l}3 \\
5\end{array}$ & $\begin{array}{l}46 / 6 \\
60 / 12\end{array}$ & $\begin{array}{l}15 / 4 \\
30 / 12\end{array}$ & $\begin{array}{l}98 \\
96\end{array}$ & $\begin{array}{l}34 \\
23\end{array}$ & $\begin{array}{l}4.4 \\
6.2\end{array}$ & $\begin{array}{l}100 \\
100\end{array}$ & $\begin{array}{l}44 \\
88\end{array}$ \\
\hline 35 & $\left\{\begin{array}{r}9 \mathrm{yr} \\
\text { I5 } \mathrm{yr}\end{array}\right.$ & $\begin{array}{l}0.94 \\
1.53\end{array}$ & $\begin{array}{r}5 \\
12\end{array}$ & $\begin{array}{l}3 \\
5\end{array}$ & $\begin{array}{l}65 / 7 \\
60 / 8\end{array}$ & $\begin{array}{l}15 / 10 \\
22 / 10\end{array}$ & $\begin{array}{l}96 \\
93\end{array}$ & $\begin{array}{l}27 \\
40\end{array}$ & $\begin{array}{l}5.2 \\
3.8\end{array}$ & $\begin{array}{r}107 \\
94\end{array}$ & $\begin{array}{l}48 \\
62\end{array}$ \\
\hline
\end{tabular}

Pressures: $\mathrm{RA}=$ right atrium $\mathrm{RV}=$ right ventricle; $\mathrm{MPA}=$ main pulmonary artery; $\mathrm{SaO}_{2}=$ arterial oxygen saturation; $\mathrm{AV} \mathrm{O}_{2}$ difference = arteriovenous oxygen difference; $Q v=$ pulmonary valve flow; $H R=$ hear rate; $S V=$ stroke volume; $S E T=$ right ventricular ejection time; VA = pulmonary valve area (+indicates area calculated from resistance).

Electrocardiogram: ( $P$ wave) $n=$ normal; $+=$ increased; $\pm=$ borderline; (mean frontal axis) $R A D=$ right axis deviation; $I=$ indeterminate axis, $n=$ normal; ( $R$ wave) $R=$ height of $R$ wave in $\mathrm{mm}$ in $V_{1}$ or $V_{4} R$.

PDA = persistent ductus arteriosus.

Conversion factor from Traditional to SI units: I $\mathrm{mmHg} \approx 0.133 \mathrm{kPa}$.

times (second study divided by first study) between I.5 and 2.0, Io had ratios from 1.05 to $\mathrm{I.26}$, and in Io the ratio ranged from 0.74 to I.O. The increases in ejection time were caused mainly by slower heart rates and older ages at the second study (Golde and Burstin, 1970).

Fig. 3A shows that calculated absolute valve orifice area rose in 20 (over $100 \%$ increase in area in 10 , from 50 to $99 \%$ increase in 5 , and under $50 \%$ increase in 5); the area was unchanged in 4 and it fell in 8 (by less than $25 \%$ in 4 and by 26 to $50 \%$ in 4). The increases in orifice area often did not keep pace with the increase in body surface area. Valve orifice area per square metre of body surface area (Fig. 3B) rose in 12 (over $100 \%$ increase in I only, 50 to $99 \%$ in 2 , and less than $50 \%$ in 9), was unchanged in 3 , and fell in 17 (by less than $25 \%$ in 9 , and more than $50 \%$ in 4 ).

Most of the striking decreases in the calculated orifice area were associated with infundibular hypertrophy.

The body surface areas of almost all the children were within normal limits and the 4 who were below the 3 rd percentile at the first study were close to the 5oth percentile at the second study. Most children grew at a normal rate, unrelated to the severity of the pulmonary stenosis, except for 2 with very severe stenosis and congestive heart failure.

The $x$-rays of the heart and chest were not helpful. None with mild or moderate stenosis had increased cardiothoracic ratios, though most had 


\begin{tabular}{|c|c|c|c|c|c|c|}
\hline \multirow[t]{2}{*}{$\operatorname{SET}(s)$} & \multicolumn{2}{|c|}{$V A\left(\mathrm{~cm}^{2}\right)$} & \multicolumn{3}{|c|}{ Electrocardiogram } & \multirow[t]{2}{*}{ Other } \\
\hline & Absolute & $\begin{array}{l}\text { per } m^{2} \\
B S A\end{array}$ & $P$ & $R$ & $\begin{array}{l}Q R S \\
\text { axis }\end{array}$ & \\
\hline 0.30 & $\begin{array}{l}0.50+ \\
0.73\end{array}$ & $\begin{array}{l}0.74+ \\
0.43\end{array}$ & $\begin{array}{l}\text { n } \\
+\end{array}$ & $\begin{array}{r}7 \\
14\end{array}$ & $\begin{array}{r}75 \\
120\end{array}$ & \\
\hline $\begin{array}{l}0.35 \\
0.30\end{array}$ & $\begin{array}{l}0.60 \\
1.25\end{array}$ & $\begin{array}{l}0.79 \\
0.88\end{array}$ & $\mathbf{n}$ & 17 & I & \\
\hline 0.30 & $\begin{array}{l}0.34+ \\
0.72\end{array}$ & $\begin{array}{l}0.45+ \\
0.61\end{array}$ & + & 12 & $\begin{array}{l}\text { RAD } \\
130\end{array}$ & Infundibular hypertrophy \\
\hline $\begin{array}{l}0.26 \\
0.26\end{array}$ & $\begin{array}{l}0.41 \\
1.80\end{array}$ & $\begin{array}{l}0.51 \\
1.30\end{array}$ & $\begin{array}{l}\mathrm{n} \\
\mathrm{n}\end{array}$ & $\begin{array}{l}18 \\
10\end{array}$ & $\begin{array}{l}135 \\
135\end{array}$ & \\
\hline 0.22 & $\begin{array}{l}0.74+ \\
0.50\end{array}$ & $\begin{array}{l}0.93+ \\
0.53\end{array}$ & $\mathbf{n}$ & 12 & IIO & Infundibular hypertrophy \\
\hline $\begin{array}{l}0.32 \\
0.40\end{array}$ & $\begin{array}{l}0.34 \\
0.40\end{array}$ & $\begin{array}{l}0.44 \\
0.31\end{array}$ & $\begin{array}{l}\text { n } \\
+\end{array}$ & $\begin{array}{l}15 \\
18\end{array}$ & $\begin{array}{l}120 \\
180\end{array}$ & \\
\hline 0.25 & 2.63 & 1.5 & $\begin{array}{l}\mathbf{n} \\
\mathbf{n}\end{array}$ & $\begin{array}{l}2 \\
6\end{array}$ & $\begin{array}{l}\mathbf{n} \\
\mathbf{n}\end{array}$ & \\
\hline 0.42 & 0.56 & 0.40 & $\begin{array}{l}\mathrm{n} \\
+\end{array}$ & $\begin{array}{r}4 \\
\text { II }\end{array}$ & $\begin{array}{r}80 \\
120\end{array}$ & \\
\hline 0.30 & $\begin{array}{l}0.70+ \\
0.70\end{array}$ & $\begin{array}{l}0.88+ \\
0.50\end{array}$ & $\mathbf{n}$ & 12 & 50 & Infundibular hypertrophy \\
\hline 0.30 & $\begin{array}{l}0.74+ \\
2.36\end{array}$ & $\begin{array}{l}0.97+ \\
1.30\end{array}$ & $\begin{array}{l}\mathbf{n} \\
\mathbf{n}\end{array}$ & $\begin{array}{l}3 \\
3\end{array}$ & 105 & \\
\hline 0.37 & $\begin{array}{l}0.65+ \\
1.09\end{array}$ & $\begin{array}{l}0.88+ \\
0.77\end{array}$ & & & & Noonan's syndrome \\
\hline $\begin{array}{l}0.35 \\
0.26\end{array}$ & $\begin{array}{l}0.56 \\
0.99\end{array}$ & $\begin{array}{l}0.60 \\
0.65\end{array}$ & $\mathbf{n}$ & 6 & 115 & \\
\hline
\end{tabular}

a cardiac contour that suggested right ventricular hypertrophy. Most had post-stenotic dilatation of the main pulmonary artery. No significant changes took place with time, even in the patients with increasing right ventricular systolic pressure. The 3 children with the severest stenosis and symptoms had right atrial and ventricular enlargement as well as an increased cardiothoracic ratio.

In the electrocardiogram, normal $P$ waves became peaked and tall in 8 patients whose right ventricular systolic pressures rose by $\mathrm{I} 4$ to $50 \mathrm{mmHg}$ ( $\mathrm{I} .9$ to $6.7 \mathrm{kPa}$ ) to reach pressures of 55 to $140 \mathrm{mmHg}$ $(7.3$ to $18.6 \mathrm{kPa})$. However, in another 6 children the $P$ waves remained normal despite 9 to 40 $\mathrm{mmHg}$ ( 1.2 to $5.3 \mathrm{kPa}$ ) rises of right ventricular systolic pressure to levels of 65 to $105 \mathrm{mmHg}$ (8.6 to $14.0 \mathrm{kPa}$ ).

In 19 children the frontal plane QRS axis was recorded at each cardiac catheterization and in another 10 it was recorded once. Most of the QRS axes were over $90^{\circ}$. In general the $Q R S$ axis and the right ventricular systolic pressure rose or fell together, but on 5 occasions one variable rose while the other fell (Fig. 4A). The height of the $R$ or $R^{\prime}$ wave in the right praecordial leads usually changed in the same direction as the change in right ventricular systolic pressure (Fig. 4B) but 6 cases were exceptions. These were not related to right ventricular conduction disturbances nor to age; the children with Noonan's syndrome are not included in these figures. 


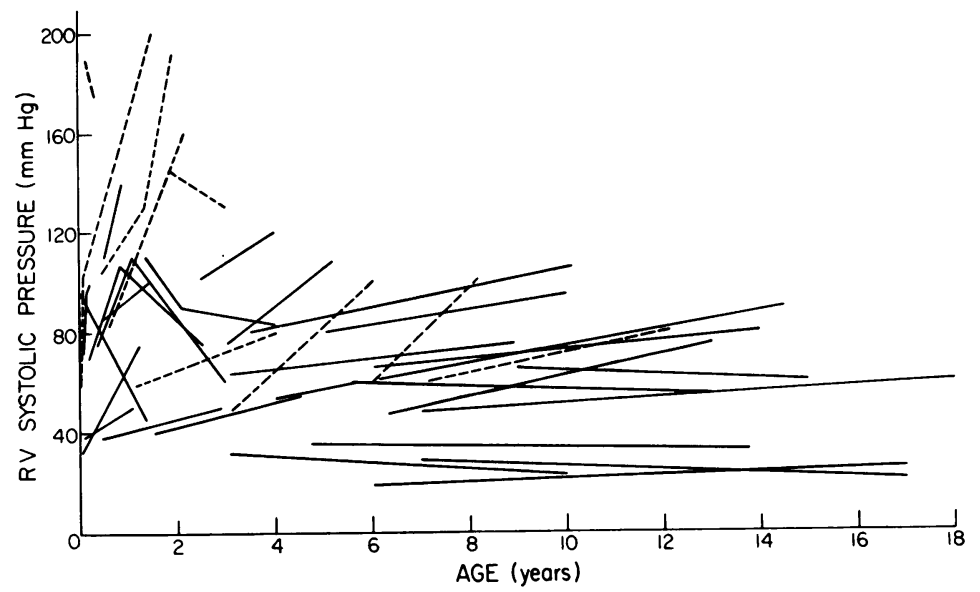

FIG. I Changes in right ventricular systolic pressure with age. The straight lines here and in subsequent figures join points in the same patient and do not indicate a linear change. Dashed lines show those with infundibular hypertrophy.

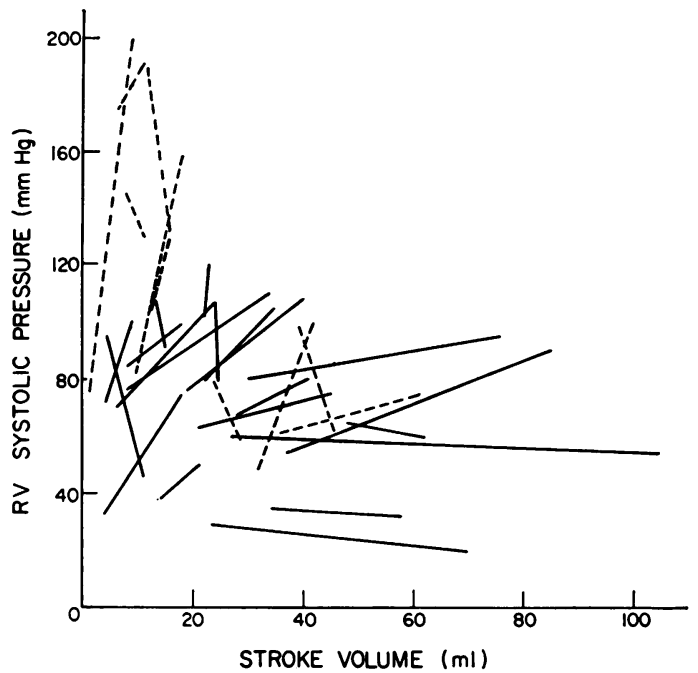

FIG. 2 Relation of stroke volume and right ventricular systolic pressure in repeat catheterizations. Dashed lines show those with infundibular hypertrophy.

\section{Discussion}

\section{Calculations}

Estimating pulmonary valve orifice area from the Gorlin formula is subject to errors in the measurement of each component of the formula, but the contribution to the total error varies for each com- ponent. The mean systolic pressure drop from right ventricle to pulmonary artery is usually measured with reasonable accuracy and, since the square root of this difference is taken, errors in the measurement have little effect on the calculated valve area. Furthermore, a high correlation between mean and peak systolic pressure difference across the pulmonary valve has been shown by Bassingthwaighte et al. (1963) and by Campbell (1960). Therefore, we believe that there was negligible error in deriving mean from peak pressure differences on the few occasions when the original tracings were not available.

Systolic ejection times are more difficult to measure at cardiac catheterization when pressures are not recorded with high fidelity. However, our repeated measurements of pressure tracings by different observers and comparisons of these measurements with phonocardiograms in 2 patients suggested that the error of measuring right ventricular systolic ejection time was no more than 20 per cent.

The major source of error lies in calculating stroke volume when oxygen uptake is not measured. Almost all the calculated high cardiac outputs were associated with low arteriovenous differences of oxygen content, however, suggesting that indeed the flows were higher than normal. Finally, increases in right ventricular pressure and right-sided forces were well correlated, so that the pressure increases were in general not caused by transiently raised stroke volumes. It is notable that many other published studies also relied on assumed oxygen consumption. 

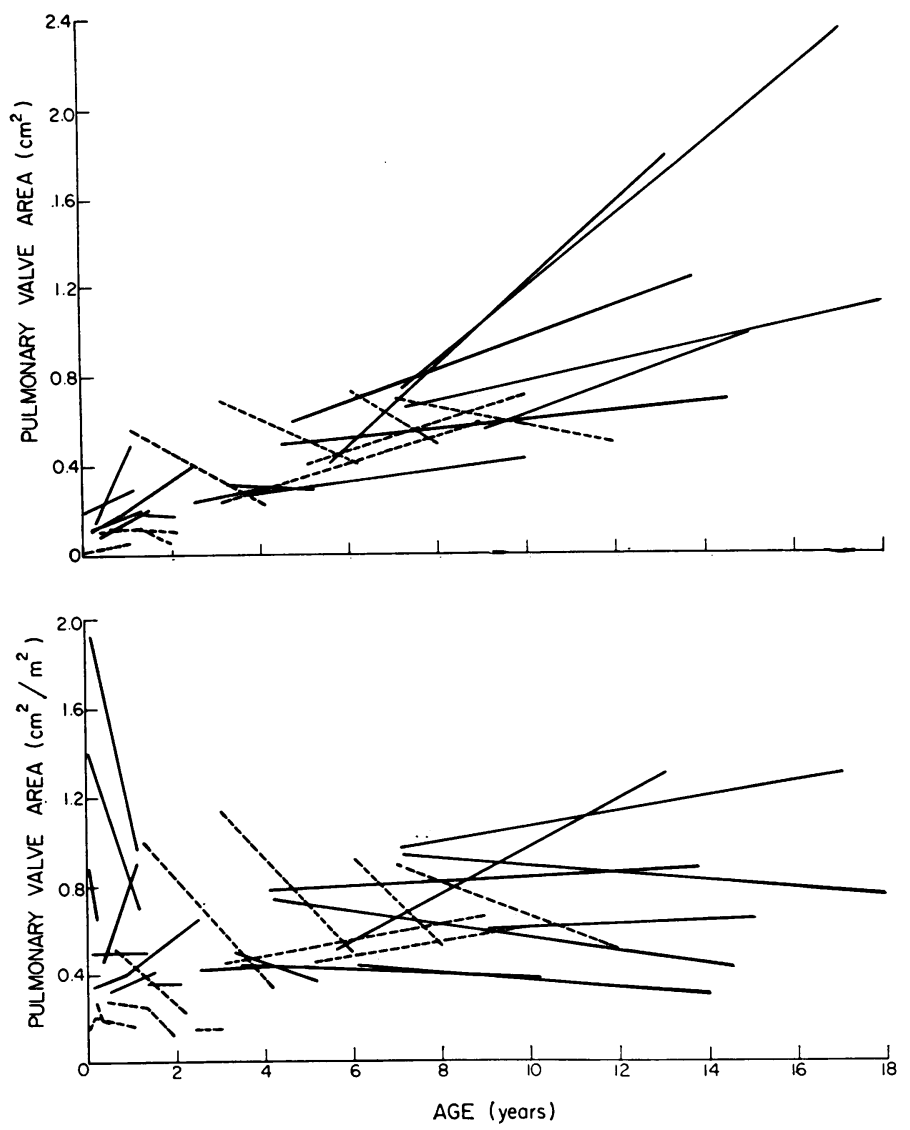

FIG. $3 A$ ) Upper panel: change in absolute pulmonary valve area (or equivalent area) with age. $B$ ) Lower panel: change in pulmonary valve area (or equivalent area) per square metre of body surface area with age. Dashed lines show those with infundibular hypertrophy.

\section{Pressure changes}

As described by Gorlin and Gorlin (1951), $\mathrm{A}=$ flow per systolic second $/ 44.5 \sqrt{ } \Delta \mathrm{P}$, where $\mathrm{A}$ is the valve orifice area and $\Delta \mathrm{P}$ is the mean systolic pressure drop from right ventricle to main pulmonary artery. Since flow per systolic second is stroke volume (SV) divided by right ventricular systolic ejection time (SET), the formula can be rearranged to give $\Delta \mathrm{P}=$ $[(\mathrm{SV} / \mathrm{SET}) / 44.5 \mathrm{~A}]^{2}$. Increases in right ventricular systolic pressure are thus caused by an increase in the ratio $[(\mathrm{SV} / \mathrm{SET}) / \mathrm{A}]^{2}$. With normal growth, cardiac output increases approximately in proportion to body surface area (Cayler, Rudolph, and Nadas, 1963; Jegier et al., 1963), though recent studies suggest a slightly better correlation with height alone (Krovetz and Goldbloom, 1972). Because of the normal slowing of heart rate from infancy to adulthood (Liebman, 1968; Walsh, 1968) stroke volume increases more rapidly than does cardiac output so that there is about a twelve- to fifteenfold increase of stroke volume from birth to adult life. At the same time, the increase in age and decrease of heart rate would be expected to increase systolic ejection time between two- and threefold (Golde and Burstin, 1970). Thus, for a fixed valve orifice area, the ratio of SV/SET would normally rise four- to sevenfold from birth to adult life, and in theory, cause sixteen- to forty-ninefold rise in mean systolic pressure difference from right ventricle to pulmonary artery. That this does not occur is because of the additional prolongation of right ventricular ejection time from the pulmonary stenosis (Vogelpoel and Schrire, I960; Gamboa, Hugenholtz, and Nadas, 1964), to increase in size of the valve orifice, or to congestive failure. In some patients with severe pulmonary stenosis right 

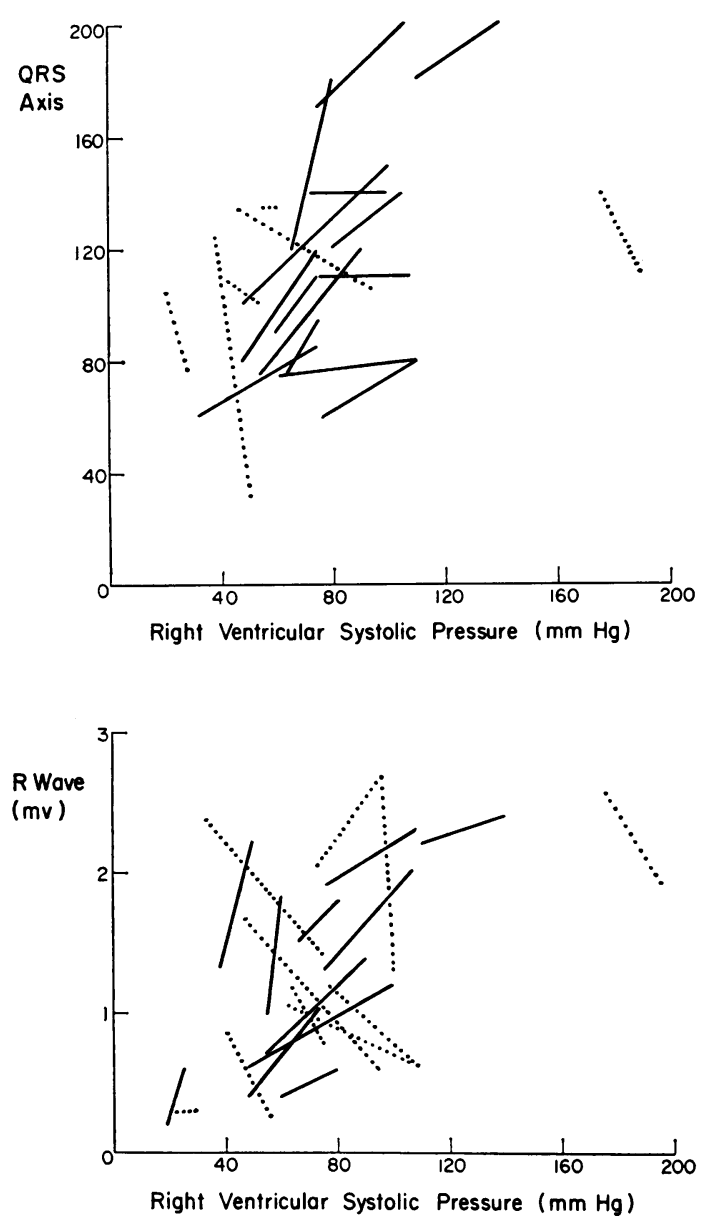

FIG. 4 Change in right ventricular systolic pressure and $A$ ) change in frontal plane $Q R S$ axis or $B$ ) change in height of $R$ or $R^{\prime}$ wave in $V_{I}$ or $V_{4} R$, whichever is largest. Dotted lines show grossly inappropriate changes.

ventricular systolic pressure does not increase much because a right-to-left atrial shunt decreases right ventricular stroke volume.

Although absolute valve orifice area usually increased with age, it seldom increased more than and often grew much less than body surface area. This suggests restricted growth of the stenotic valve, and confirms the hypothesis of Engle and Taussig (1950). Moller and Adams (1965) and Lueker et al. (1970) found valve area to increase in proportion to body surface area; however, their patients were older and did not show increases in right ventricular systolic pressure. Absolute decreases of calculated orifice area seemed to be caused usually by infundi- bular hypertrophy rather than by narrowing of the valve orifice. In our small series, severe infundibular hypertrophy occurred in patients before 2 years of age; in these children right ventricular systolic pressures were initially over $70 \mathrm{mmHg}$ $(9.3 \mathrm{kPa})$. However, one boy at 3 years of age had mild infundibular hypertrophy with a right ventricular systolic pressure of $48 \mathrm{mmHg}(6.4 \mathrm{kPa})$; by 6 years of age the pressure had risen to $100 \mathrm{mmHg}$ (13.3 $\mathrm{kPa})$ and there was severe infundibular hypertrophy.

The results of our study show that right ventricular pressures often increased, particularly in young children, and that in young children the chance of increase was independent of initial pressure levels (Table 2A). By contrast, analysis of published cases suggests that increases in pressure are uncommon and occur predominantly in those over 5 years old when first catheterized (Table 2B). Consequently, either the existing reports give too optimistic a prognosis or our data err in being too pessimistic. One of the difficulties in deciding between these alternatives is that there is considerable selection involved. It is likely that many children of all ages with clinically mild and stable pulmonary stenosis are not catheterized or recatheterized. On

TABLE 2 Numbers of children arranged by age and right ventricular systolic pressure at first cardiac catheterization

\begin{tabular}{|c|c|c|c|c|}
\hline \multirow{2}{*}{\multicolumn{2}{|c|}{$\begin{array}{l}\text { Initial right ventricular } \\
\text { systolic pressure }(\mathrm{mmHg})\end{array}$}} & \multicolumn{3}{|c|}{ Age at first study (yr) } \\
\hline & & $<5$ & 5 to 15 & $>15$ \\
\hline \multicolumn{5}{|l|}{ A } \\
\hline \multirow{5}{*}{$\begin{array}{l}50 \text { or less } \\
51 \text { to } 75 \\
76 \text { to } 100 \\
\text { 101 or more }\end{array}$} & 6.7 or less & $7(2)$ & $4(1)$ & \\
\hline & $6.8-10.0$ & $6(4)$ & $5(2)$ & \\
\hline & $10.1-13.3$ & $6(3)$ & $I(0)$ & \\
\hline & 13.4 or more & $6(2)$ & $0(0)$ & \\
\hline & & 25 (II) & I0 (3) & \\
\hline \multicolumn{5}{|l|}{ B } \\
\hline 50 or less & 6.7 or less & $2(0)$ & 19 (I) & $8(0)$ \\
\hline 51 to 75 & $6.8-10.0$ & $9(0)$ & $29(2)$ & $5(0)$ \\
\hline 76 to 100 & $10.1-13.3$ & $2(0)$ & $12(5)$ & $3(2)$ \\
\hline \multirow[t]{2}{*}{ ror or more } & I3.4 or more & $2(I)$ & $9(3)$ & $5(1)$ \\
\hline & & I5 (I) & 69 (II) & 2I（3） \\
\hline
\end{tabular}

Numbers in parentheses indicate those whose pressures had risen $20 \mathrm{mmHg}(2.7 \mathrm{kPa})$ or more at the second study. $A$ ) Data from present study. $B$ ) Data from reported studies (Fabricius, 1959; Engle et al., 1964; Tinker et al., 1965; Levine and Blumenthal, 1965; Moller and Adams, 1965; Loogen et al., 1966; Lueker et al., 1970; Møller et al., 1973). Figures in italics are pressures in $\mathbf{~ P a}$. 
the other hand, children who have high right ventricular systolic pressures when young either die or are operated on, and so would not appear in the later phases of any natural history study. Furthermore, it is only relatively recently that serial catheterizations in infants with pulmonary stenosis have been reported; there are 13 first studied under I year of age in our series, and none in the series collected from the published reports. While a randomized, prospective study will be necessary to define the risk of increasing severity of pulmonary stenosis at each stage and initial pressure, it is clear from our data that significant increases may be commoner in very young children than is presently reported.

While our series does not give accurate estimates of the risk of increases in severity of pulmonary stenosis, it does allow some prognostication. An infant with a moderately high or high right ventricular systolic pressure caused by pulmonary stenosis has a small absolute valve orifice area, with a valve that is probably thick and, therefore, not likely to grow adequately; with growth and increase of stroke volume the pressure is likely to rise considerably, and the rise will be accentuated if infundibular stenosis develops. The older child with only slightly raised right ventricular systolic pressure has at that time a reasonably large valve orifice, the valve is probably only slightly thickened and thus perhaps capable of growing well, and there will be relatively little further increase of stroke volume.

The factors that cause the right ventricular pressure to rise or remain constant appear to be similar in those with typical valvar pulmonary stenosis and in those with added mild peripheral pulmonary arterial stenosis or even those with Noonan's syndrome. One child with Noonan's syndrome had an orifice area that kept pace with body growth, so that right ventricular pressure rose only slightly from 7 to 18 years, while the other, who had a myxomatous valve, developed a rapid and striking rise of right ventricular systolic pressure related mainly to the development of conspicuous infundibular hypertrophy at an early age.

Since it is not yet possible to predict the course of this lesion in young children, they should be followed carefully to determine whether cardiac catheterization is needed or should be repeated. Body growth is normal (Fabricius, 1959) and the children are usually asymptomatic unless the stenosis is extremely severe. Radiological changes are not sensitive indicators of early increases of severity. The phonocardiogram is likely to be a better method of following the severity of pulmonary stenosis (Vogelpoel and Schrire, I960; Gamboa et al., 1964), though no one has confirmed this, especially in very young children. It is also pos- sible that the echocardiogram may eventually prove a useful tool. At present, however, the most commonly used indirect method of assessing the severity of pulmonary stenosis is the electrocardiogram (Cayler, Ongley, and Nadas, 1958; Fabricius, 1959; DePasquale and Burch, 1960; Bassingthwaighte et al., 1963; Engle and Goldberg, 1964; Hugenholtz and Gamboa, 1964), since in pulmonary stenosis there is a good correlation between the height of the $R$ wave in the right chest leads $\left(\mathrm{VI}_{1}\right.$ or $\left.\mathrm{V}_{4} \mathrm{R}\right)$ and the right ventricular systolic pressure. Usually, however, these results have not been obtained by serial studies. Furthermore, despite the good correlation coefficients, the variability about the regression line is considerable and usually not commented on. As a result of this variability, the prediction of right ventricular systolic pressure from the height of the $\mathbf{R}$ wave in VI or V4R is subject at times to large errors; the important error of missing severe or increasing stenosis because it is not reflected in the electrocardiogram has been stressed by Boyle, Morton, and Pantridge (1964). Our results of serial studies in the same patient confirm not only the tendency for the height of the $R$ wave to reflect the right ventricular systolic pressure but also the variability of this relation and the possibility of missing an increase in severity by relying only on the electrocardiogram.

\section{References}

Abrahams, D. G., and Wood, P. (1951). Pulmonary stenosis with normal aortic root. British Heart fournal, 13, 519.

Bassingthwaighte, J. B., Parkin, T. W., DuShane, J. W., Wood, E. H., and Burchell, H. B. (1963). The electrocardiographic and hemodynamic findings in pulmonary stenosis with intact ventricular septum. Circulation, $28,893$.

Benton, J. W., Jr., Elliott, L. P., Adams, P., Jr., Anderson, R. C., Hong, C. Y., and Lester, R. G. (1962). Pulmonary atresia and stenosis with intact ventricular septum. American fournal of Diseases of Children, 104, 161.

Boyle, D. McC., Morton, P., and Pantridge, J. F. (1964). The electrocardiogram in pulmonary valve stenosis with intact ventricular septum. British Heart fournal, 26, 477.

Campbell, M. (1960). Relationship of pressure and valve area in pulmonary stenosis. British Heart fournal, 22, 101.

Campbell, M., and Missen, G. A. K. (1959). Survival in good health until 65 years with pulmonary valvar stenosis. Guys Hospital Reports, 108, 390.

Carlgren, L. E. (1959). Incidence of congenital heart disease in children born in Gothenburg 1941-1950. British Heart Fournal, 21, 40.

Cayler, G. G., Ongley, P., and Nadas, A. S. (1958). Relation of systolic pressure in the right ventricle to the electrocardiogram. A study of patients with pulmonary stenosis and intact ventricular septum. New England fournal of Medicine, 258, 979.

Cayler, G. G., Rudolph, A. M., and Nadas, A. S. (1963). Systemic blood flow in infants and children with and without heart disease. Pediatrics, 32, 186.

DePasquale, N., and Burch, G. E. (1960). The electrocardiogram and ventricular gradient in isolated congenital pulmonary stenosis. Circulation, 21, 181 . 
Engle, M. A., Ito, T., and Goldberg, H. P. (1964). The fate of the patient with pulmonic stenosis. Circulation, 30, 554 .

Engle, M. A., and Taussig, H. B. (1950). Valvular pulmonic stenosis with intact ventricular septum and patent foramen ovale. Circulation, 2, 481.

Fabricius, J. (1959). Isolated Pulmonary Stenosis. Munksgaard, Copenhagen.

Gamboa, R., Hugenholtz, P. G., and Nadas, A. S. (1964). Accuracy of the phonocardiogram in assessing severity of aortic and pulmonic stenosis. Circulation, 30, 35 .

Gersony, W. M., Bernhard, W. F., Nadas, A. S., and Gross, R. E. (1967). Diagnosis and surgical treatment of infants with critical pulmonary outflow obstruction. Circulation, $35,765$.

Golde, D., and Burstin, L. (1970). Systolic phases of the cardiac cycle in children. Circulation, 42, 1029.

Golinko, R. J., and Rudolph, A. M. (I96I). A valve for respiratory studies in infants. Pediatrics, 27, 645.

Gorlin, R., and Gorlin, S. A. (195I). Hydraulic formula for calculation of the area of the stenotic mitral valve, other cardiac valves, and central circulatory shunts. I. American Heart fournal, 41, r.

Hoffman, J. I. E., and Rudolph, A. M. (1965). Natural history of ventricular septal defects in infancy. American fournal of Cardiology, 16, 634.

Hugenholtz, P. G., and Gamboa, R. (1964). Effect of chronically increased ventricular pressure on electrical forces of the heart. Circulation, 30, 5 I I.

Jegier, W., Sekelj, P., Auld, P. A. M., Simpson, R., and McGregor, M. (1963). The relation between cardiac output and body size. British Heart fournal, 25, 425 .

Johnson, A. M. (1959). Hypertrophic infundibular stenosis complicating simple pulmonary valve stenosis. British Heart fournal, 21, 429.

Kirklin, J. W., Connolly, D. C., Ellis, F. H., Jr., Burchell, H. B., Edwards, J. E., and Wood, E. H. (1953). Problems in the diagnosis and surgical treatment of pulmonic stenosis with intact ventricular septum. Circulation, 8, 849.

Krovetz, L. J., and Goldbloom, S. (1972). Normal standards for cardiovascular data. I. Examination of the validity of cardiac index. Fohns Hopkins Medical fournal, 130, 174.

LaFarge, C. G., and Miettinen, O. S. (1970). The estimation of oxygen consumption. Cardiovascular Research, 4, 23.

Lambert, E. C., Canent, R. V., and Hohn, A. R. (1966). Congenital cardiac anomalies in the newborn. A review of conditions causing death or severe distress in the first month of life. Pediatrics, 37, 343.
Levine, O. R., and Blumenthal, S. (1965). Pulmonic stenosis. Circulation, 32, Suppl. III, 33.

Liebman, J. (1968). Electrocardiography. In Heart Disease in Infants, Children and Adolescents, p. 183. Ed. by A. J. Moss and F. H. Adams. Williams and Wilkins, Baltimore.

Little, J. B., Lavender, J. P., and DeSanctis, R. W. (1963). The narrow infundibulum in pulmonary valvular stenosis. Circulation, 28, 182.

Loogen, F., Gleichmann, W., and Wilke, K. H. (1966). Verhalten der Pulmonalstenose während des Wachstums. Zeitschrift für Kreislaufforschung, 55, II64.

Lueker, R. D., Vogel, J. H. K., and Blount, S. G., Jr. (1970). Regression of valvular pulmonary stenosis. British Heart fournal, 32, 779.

Luke, M. J. (1966). Valvular pulmonic stenosis in infancy. fournal of Pediatrics, 68, 90.

Moller, I., Wennevold, A., and Lyngborg, K. E. (1973). The natural history of pulmonary stenosis. Cardiology, 58, 193

Moller, J. H., and Adams, P. (1965). Natural history of pulmonary valvular stenosis. Serial cardiac catheterizations in 21 children. American fournal of Cardiology, 16, 654 .

Moller, J. H., and Adams, P., Jr. (I966). A simplified method for calculating the pulmonary valvular area. American Heart fournal, 72, 463.

Rowe, R. D., and Cleary, T. E. (1960). Congenital cardiac malformation in the newborn period; frequency in a children's hospital. Canadian Medical Association fournal, 83, 299.

Rudolph, A. M., and Cayler, G. G. (1958). Cardiac catheterization in infants and children. Pediatric Clinics of North America, 5, 907.

Selzer, A., Carnes, W. H., Noble, C. A., Jr., Higgins, W. H., Jr., and Holmes, R. O. (1949). The syndrome of pulmonary stenosis with patent foramen ovale. American Fournal of Medicine, 6, 3 .

Tinker, J., Howitt, G., Markman, P., and Wade, E. G. (1965). The natural history of isolated pulmonary stenosis. British Heart fournal, 27, 151.

Vogelpoel, L., and Schrire, V. (1960). Auscultatory and phonocardiographic assessment of pulmonary stenosis with intact ventricular septum. Circulation, 22, 55.

Walsh, S. Z. (1968). Disorders of heart rhythm and their treatment. In Paediatric Cardiology, p. 882. Ed. by $\mathbf{H}$. Watson. Lloyd-Luke, London.

Requests for reprints to Professor Julien I. E. Hoffman, Department of Pediatrics, University of California, San Francisco, California 94143, U.S.A. 